\title{
ESTIMATION OF TECHNOGENIC DANGER RISK OF TECHNICO- ECONOMIC SYSTEMS
}

\author{
Nikolay Petrov \\ Technical University - Sofia
}

Keywords: risk; danger of technogenic; technico-economic systems; func-tionnal elements

Summary: The article considers the study of technogenic danger risk of technico-economic systems in daily operation on the part of a human operator. In the techno-economic systems consist of a specified quantity of functional elements, United parallel or sequentially.

Introduction: The risk as an event or set of events, holds a variety of discrete and/or ongoing implementations, each of which has its likelihood of occurrence and the amount of damage. The risk in modern science, culture and society is the summary category as sin, justice, value, meaning, etc. (Ivan Poptchev, Strategies for managing the risk of NBU, Sofia, 2004, p. 5, p. 6) [1].

Under the techno-economic system (TES) is a system of interacting manufacturing engineering (technology) equipment for automation and human operator [2]. The refusal of the FE of the TES is the reason for the technogenic danger (risk), continued throughout the period of technical operation (TO) of the systems [4].

For example, on the aircraft during flight is possible to be denied imposing performance of repair of the service crew, if the refused is not reserved with the FE upright one. In addition, the need to increase the technical resource of aircraft requires the placement of new technological systems (connected in parallel or in sequence of old), compatible with the available standard systems installed by the manufacturer.

Similarly, is the State with the TES of a pipeline, the composite FE (units of pipelines) that pass through the village. It should be borne in mind that the maximum level of probability of failure of arbitrary complex TES is $10^{-9}$, i.e. on a billion connections in nature one of them failed [2].

Exposure: the possible statuses of the TES are: all their constituent FE are in order and the system does not represent a technogenic danger; at least one composite FE refused and the system is in a State of repair, i.e. represents the technogenic danger.

Since these conditions occur accidentally, the following questions arise: what are the estimates of the probability of FE of the TES to be in such a State at any point of time $t \quad(0<t<\infty)$; what are the marginal ratings of these pro-babilities for large times $(t \rightarrow \infty)$ and small times $(t \rightarrow 0)$ of exploitation. The decision on the first question was committed in work [3]. This paragraph is dedicated to resolving the second question.

Each TES can be presented as composed of parallel or sequential United nodes (the node is unrecovered FE). At the beginning of the study will determine the marginal probability assessments for safe and unsafe condition of components with parallel United FE (there are other types of compounds, which, however, are not the subject of this review).

\section{Parallel connected FE of TES}

For clarity of argument, it is considered that the constituent parts of the TES consists of two parallel United FE. The two FE are assumed to have constant intensity of flow of rejections, respectively $\Lambda_{1}>0$ for the first FE and $\Lambda_{2}>0$ for the second. As a component part of the TES store its working capacity, the unification of the FE assumes that is "loaded", i.e. is 
present"hot" reservation. Simultaneous failure of both FE is excluded. In these conditions is determined the intensity of the stream of rejections $\omega(t)$ the component part of the system [6]:

$$
\omega(t)=\frac{\Lambda_{1}+\Lambda_{2} \exp \left[-\left(\Lambda_{2}-\Lambda_{1}\right) t\right]-\left(\Lambda_{2}+\Lambda_{1}\right) \exp \left(-\Lambda_{2} t\right)}{1+\exp \left[-\left(\Lambda_{2}-\Lambda_{1}\right) t\right]-\exp \left(-\Lambda_{2} t\right)}
$$

If the intensity of the refunds on any FE of the components of the TES is constant and equal to $M_{1}>0$ for the first FE and $M_{2}>0$ for the second FE $\left(M_{1}>M_{2}\right)$, the intensity of the refunds of the component part will be determined by the [6]:

$$
\mu(t)=\frac{M_{1} M_{2}\left\{1-\exp \left[-\left(M_{2}-M_{1}\right) t\right]\right\}}{\left\{M_{2}-M_{1} \exp \left[-\left(M_{2}-M_{1}\right) t\right]\right\}} .
$$
by:

Since the probability of a safe condition $P_{S C}(t)$ the component part of the TES is defined

$$
P_{S C}(t)=1-P_{D C}(t)
$$

where $P_{D C}(t)$ is the probability of dangerous condition (DC) of the TES in the same moment of time.

From formulas (1) to (3) follows the following differential equation

$$
\frac{d P_{S C}(t)}{d t}+[\omega(t)+\mu(t)] P_{S C}(t)=\mu(t)
$$

Equation (4) is decided in the performance of the condition:

$$
P_{S C}(0)=1 \text {, }
$$

corresponding to a safe condition at the beginning of the operation $(t=0)$.

The decision of (4) is determined by the following formula:

where

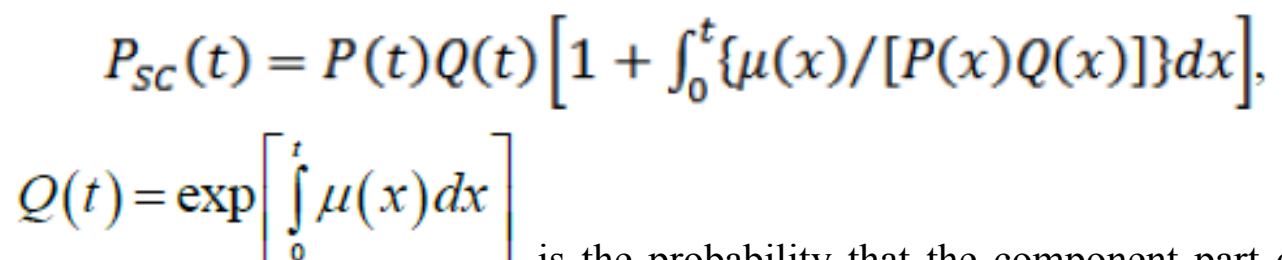
is the probability that the component part of the TES to be recovered in the course of time $t ; P(t)$ - the probability for a faultless operation of the component part in the passage of time $t$.

Lets consider the marginal probability assessments for safe and dangerous (risky) status of the component part at a great value $(t \rightarrow \infty)$ and small value $(t \rightarrow 0)$ of operation time.

$$
\lim _{t \rightarrow \infty} P_{S C}(t)=M /(\Lambda+\mathrm{M})
$$

where

$$
\Lambda=\lim _{t \rightarrow \infty} \lambda(t) ; M=\lim _{t \rightarrow \infty} \mu(t) \text {. }
$$

According formulas (1) and (2) follow the expressions $\lim _{t \rightarrow \infty} \lambda(t)=\Lambda_{1}, \lim _{t \rightarrow \infty} \mu(t)=M_{1}$. By the use of formulas (3) and (7) is obtained: 


$$
\lim _{t \rightarrow \infty} P_{S C}(t)=K_{C R}^{(1)}, \lim _{t \rightarrow \infty} P_{D C}(t)=K_{C S}^{(1)},
$$

where $K_{C R}^{(1)}=M_{1} /\left(\Lambda_{1}+M_{1}\right)_{\text {is }}$ coefficient of readiness(CR) of the first $\mathrm{FE}$; $K_{C S}^{(1)}=\Lambda_{1} / \Lambda_{1}+M_{1}$ is coefficient of stay (CS) of the same FE.

From (8) follows the formula of technogenic danger (TD) risk:

$$
R_{T D}(\Delta t)=P_{D}(\Delta t) \cdot Z_{T D}(\Delta t)=K_{S}^{(1)} \cdot Z_{T D}(\Delta t),
$$

where: $Z_{T D}(\Delta t)$ are economic losses from the relative occurrence of techno-genic danger (TD) (to gain on the normal function of the TES); $\Delta t=t_{2-} t_{1}$.

Thus, the constituent parts of the TES with parallel laden connection of FE, the limit fixed value of the probability of a safe condition $P_{S C}(t)$ at $t \rightarrow \infty$ strives to the coefficient of readiness $K_{C R}$ of the most reliable FE. At the same time, the limit fixed value of the probability of a dangerous condition $P_{D C}(t)$ is strives to the coefficient of stay $K_{C S}$ of the most reliable FE.

Similarly is the solution of the task provided that the intensity of the denials of the FE of the components of the TES are identical, i.e. $\Lambda_{1}=\Lambda_{2}=\Lambda$, where $\Lambda>0$ is a constant. Such a combination of FE in reliability theory is known as "hot" reservation of basic (first) FE. In this case, the intensity of the denials of the components of the TES will have the form:

$$
\omega(t)=2 \Lambda[1-\exp (-\Lambda t)] /[2-\exp (-\Lambda t)]
$$

Accordingly, the intensity of the recovery is:

$$
\mu(t)=M^{2} t /(1+M t),
$$

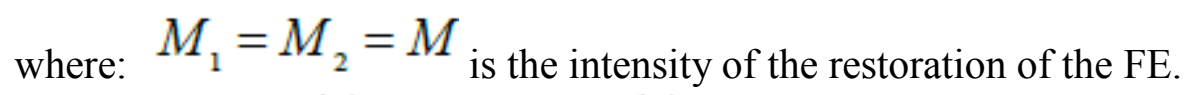

$$
\text { Since } \lim _{t \rightarrow \infty} \lambda(t)=\Lambda, \lim _{t \rightarrow \infty} \mu(t)=M \text {, it under (7) thies are determined: }
$$

$$
\lim _{t \rightarrow \infty} P_{S C}(t)=K_{C R}, \quad \lim _{t \rightarrow \infty} P_{D C}=K_{C S},
$$

where: $\quad K_{C R}=M /(\Lambda+\mathrm{M})$ is the coefficient of readiness of the first $\mathrm{FE}$; $K_{C S}=\Lambda / \Lambda+\mathrm{M}$ is the coefficient of stay of this FE.

Therefore, the component part of the TES with "hot" reservation, the basic FE has limit value of the probability of safe (risk-free) status (at $t \rightarrow \infty$ ), striving to $K_{C R_{\text {On }}}$ the primary node. Accordingly, the limit fixed value of the proba-bility of a dangerous condition is determined by the coefficient of this FE.

The limit values of the likelihood of a safe condition $P_{S C}(t)$ and the pro-bability of a dangerous condition $P_{D C}(t)$ of the TES at $\tau \rightarrow \infty$ are determined from $\lim _{t \rightarrow 0} P_{S C}(t)=1$ and $\lim _{t \rightarrow 0} P_{D C}(t)=0$. From these limit ratios, formula (3) and the Maclaurin expansion at $t \rightarrow 0$ follows:

$$
P_{S C}(t)=1-A t^{\alpha}+o\left(t^{\alpha}\right) ; \quad P_{D C}(t)=A t^{\alpha}+o\left(t^{\alpha}\right),
$$


where $A, a$ are positive constants; $O\left(t^{\alpha}\right)$ - infinitely small quantity of a higher order of magnitude than $t^{\alpha}$.

Here the question arises what are the values of $A$ and $\alpha$ in a limit case, i.e. at $t \rightarrow 0$. In this connection, we prove the following theorem.

Theorem 1. If the constituent parts of the TES contain two parallel charged FE, the intensity of the refusals, which are $\Lambda_{1}>0$ and $\Lambda_{2}>0$ for the probability of safe and dangerous state of its constituent parts will be fair the following ratios:

$$
\lim _{t \rightarrow 0}\left[1-P_{S C}(t)\right] t^{-2}=\Lambda_{1} \Lambda_{2} ; \lim _{t \rightarrow 0} P_{D C}(t) t^{-2}=\Lambda_{1} \Lambda_{2}
$$

Before the proof of the theorem is noticeable that by formula (13) in the $t \rightarrow 0$ derive the following estimates of the probability of safe and dangerous condition of the components:

$$
P_{S C}(t)=1-\Lambda_{1} \Lambda_{2} t^{2}+o\left(t^{2}\right) ; \quad P_{D C}(t)=\Lambda_{1} \Lambda_{2} t^{2}+o\left(t^{2}\right) .
$$

Therefore, when $t \rightarrow 0$ follow relations: $A=\Lambda_{1} \Lambda_{2}, \alpha=2$.

Proof: The proof of Theorem 1 is done by expansion in series of Mac-laurin of the likelihood of a safe condition $P_{S C}(t)$ the component part of the TEC. The decomposition is performed in the vicinity of point in degrees current time, by limiting its quadratic part. Moreover, if the $t \rightarrow 0$ follows:

$$
P_{S C}(t)=P_{S C}(0)+\dot{P}_{S C}(0) \cdot t+0,5 \ddot{P}_{S C}(0) \cdot t^{2}+o\left(t^{2}\right) \text {. }
$$

In accordance with the formulae (1) and (2) it follows that $\omega(0)=0 ; \mu(0)=0$. Taking into account the above fact and (5) in the ratio of differential equation (4) is determined $\dot{P}_{S C}(0)=0$.

By the recording of (4.5.5) and (4.5.15) follows:

$$
P_{S C}(t)=1+0,5 \ddot{P}_{S C}(0) \cdot t^{2}+o\left(t^{2}\right) \text {. }
$$

For the determination of the value of the $\ddot{P}_{S C}(0)$ using differential equa-tion (4), which follows:

$$
\ddot{P}_{S C}(0)=\dot{\mu}(t)-[\dot{\omega}(t)+\dot{\mu}(t)] P_{S C}(t)-[\omega(t)+\mu(t)] \dot{P}_{S C}(t) .
$$

From equation (1) follows $\dot{\omega}(0)=2 \Lambda_{1} \Lambda_{2}$, and from (17) follows equation $\ddot{P}_{S C}(0)=-2 \Lambda_{1} \Lambda_{2} \cdot$ Put the last expressions in (16) and receiving the first formula (13). Using (3) gets the second proof (14). This completes the proof of Theorem 1.

By guest on (14) it is noticeable that at small times of operation (valid especially for the aircrafts), the probability of safe and dangerous condition of the constituent parts of the TES (having parallel laden combination of FE) only depends on the intensity of the refusals of FE and does not depend on the intensity of the restoration. The physical nature of this phenomenon lies in the fact that in the home stretch of the exploitation of the constituent parts of the system reliability of FE property prevails and not the reimbursement of work ability. 


\section{Consistently United FE of the TEC}

The overall assessment of the likelihood of safe and dangerous condition of the TES is done as for the simplicity of the argument it is assumed that the component consists of two successive United FE. The denials of FE are independent of each other and have a constant intensity. The intensity of the restoration of the composite part is constant and equal to $M>0$. It follows the following theorem.

Theorem 2. Let the component part of the TES to contain two consecutively United FE that the intensity of the stream of rejections is accordingly $\Lambda_{1}>0$ and $\Lambda_{2}>0$. Then the probability of safe and dangerous condition of the component part will be fair the ratios:

$$
\lim _{t \rightarrow 0}\left[1-P_{S C}(t)\right] t^{-1}=\Lambda_{1}+\Lambda_{2} ; \lim _{t \rightarrow 0} P_{D C}(t)=\Lambda_{1}+\Lambda_{2}
$$

Proof: In accordance with formulas (6) and (3) the determination of the following expressions:

$$
\begin{gathered}
P_{S C}(t)=[M+\Lambda P(t) Q(t)] /(\Lambda+M), \\
P_{D C}(t)=\Lambda[1-P(t) Q(t)] /(\Lambda+M),
\end{gathered}
$$

where: $P(t)=\exp (-\Lambda t)$ is the probability of faultless operation of the component part of the TES in the course of time $t ; Q(t)=\exp (-M t)$-possibility for the component part in the passage of time $t$ not to be restored.

In the formula (4.5.19) the intensity of the stream of rejections $\Lambda$ the component is determined by our famous $\Lambda_{1}>0$ and $\Lambda_{2}>0$ of FE, according:

$$
\Lambda=\Lambda_{1}+\Lambda_{2} \text {. }
$$

As in the performance of the condition $t \rightarrow 0$ formulas are valid:

$$
P(t)=1-\Lambda t+o(t) \text { and } Q(t)=1-M(t)+o(t)
$$

by replacing $P(t)$ and $Q(t)$ in (19) are determined formulas (18), which finishes the proof of Theorem 2.

From formula (4.5.18) derives the probability for evaluation safe and dangerous condition of the components of the TES provided $t \rightarrow 0$ :

$$
P_{S C}(t)=1-\left(\Lambda_{1}+\Lambda_{2}\right) t+o(t), P_{D C}(t)=\left(\Lambda_{1}+\Lambda_{2}\right) t+o(t)
$$

Formula (4.5.22) shows that the marginal ratings do not depend on the intensity of the restoration of the TES.

By theorem 2, you receive the following:

Consequence: If the component part of the TES contains two identical FE, United with each other, alternately, for its likelihood of safe and dangerous condition are fair ratios:

$$
\lim _{t \rightarrow 0}\left[1-P_{S C}(t)\right] t^{-1}=2 \Lambda_{0} ; \lim _{t \rightarrow 0} P_{D C}(t) t^{-1}=2 \Lambda_{0}
$$

where: $\Lambda_{0}>0$ is the intensity of FE-refusals.

For fixed values of the probability of safe and dangerous condition of the components of the TES at $t \rightarrow \infty$ from the formulas (4.5.11) follows:

$$
\lim _{t \rightarrow \infty} P_{S C}(t)=K_{C R}^{(C P)}, \lim _{t \rightarrow \infty} P_{D C}(t)=K_{C S}^{(C P)},
$$


where $K_{C R}^{(C P)}=M /(\Lambda+M)$ is coefficient of readiness (CR) of composite part (CP) of TES; $K_{C S}^{(C P)}=\Lambda /(\Lambda+M)$ is coefficient of stay (CS) of the composite part (CP) of TES; $\Lambda_{\text {- is }}$ the value of intensity flow of refusals, determined by formula (20).

Therefore, the limit fixed value of the probability of a safe state of the components of considered TIS with consistently united FE at $t \rightarrow \infty$ strive for the preparedness coefficient of component part. Limit fixed value of the probability of a dangerous state of the components of the TIS strives for the stay coefficient of this part.

At the same time by circulating formula (24), it follows that the mathe-matical expressions $\lim _{t \rightarrow \infty} P_{S C}(t)=1, \lim _{t \rightarrow \infty} P_{D C}(t)=0$, are fair for each of the current value of the parameter $t$.

\section{CONCLUSION}

The result of the study of dangerous and safe condition of reimbursable TES is created with a method implementation in technical exploitation of the air force and civil aviation [6]. Proven are two theorems and are possible the following generalizations:

1. At small times of operation (valid for aircrafts) relative to the total technical resource, the likelihood of safe and dangerous condition of the constituent parts of the studied systems (with parallel laden combination of elements) depends only on the intensity of the failures of the same and does not depend on the intensity of the restoration.

2. The fixed value of the probability for the safe condition of the TEC, with parallel laden connection of items in continuous service aspires to the coefficient of the readiness of most reliable item.

3. The limit fixed value of the probability for the safe condition of the TES, with a consistently united functional elements in continuous service strives to the coefficient of the readiness of its main (base) part.

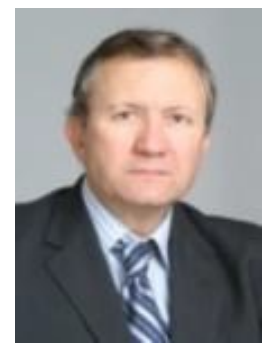

Nikolay Ivanov Petrov received M. Sc. Degree at the National University "V. Levski" - V. Tarnovo, Aviation Faculty. On 10 January 1996 he has been conferred on $\mathrm{PhD}$ by dissertation work on the topic "Synthesis of functional and stochastic methods and systems for diagnostics and repair of air radio electronics and control and measurement installations".

On 10 April 2002 he has been conferred on Dr.Sc. by defense of the dissertation on the topic "Optimization and management of the technical exploitation of air systems". On 01.10.2004 he has defended successfully diploma project for a master on "Economics" at University for National and World Economy - Sofia.

In 2007 he was awarded Professor of the Trakia University on specialty „Atomized Systems for Treatment of Information and Control". He has more than 400 scientific works, publications and developments, 100 of which - abroad. He has published 35 scientific books and textbooks, 15 of which - monographs. 
On 29 September 2009 he is chosen as Professor at Technical University, Sofia. On 29.07.2011 he was awarded a diploma for specialization "Philosophy of natural science problems" in the University "Prof. D-r Asen Zlatarov", Burgas. In 2012, during his participation in the Contest for academician correspondent of Academic corresponding member of the Bulgarian Academy of Sciences he was ranked sixth place.

Professor Petrov has been taking part in many scientific congresses and conferences, for example in Miami (USA), Vienna (Austria), Sankt Petersburg (Russia), Athens (Greece), Brno (Czech), Kiev and Lvov (Ukraine), Istanbul (Turkey), Lodz (Poland) and others.

Contact

Nikolay Iv. Petrov, Prof., Dr.Sc., 1784 Sofia, Poligona Str., № 8, Fl. 70;

Phone: +359-2-974-46-91, 0887/338-978

\section{References}

[1]. Popchev, Ivan. Strategies for risk management. NEW BULGARIAN UNIVERSITY, Sofia, Bulgaria, 2004, p. 5, p. 6.

[2]. Popchev Ivan. Six themes and literature about risk management. NBU, 2012.

[3]. Gindev, E. Fundamentals of applied reliability. Part 1. Academic ed. Prof. Marin Drinov, Sofia, 2000.

[4]. Petrov, N., Theory of catastrophes and the risk. TU, 2011

[5]. Petrov, N., N. Atanasov. Probability and Orderlines is the Nature. IJMSEA, v. 7, № III, 2013, pp.287-293.

[6]. Petrov, N. Reliability researches of technical systems at risk. Monograph. TU-Sofia, Second Edition, 2009. 